\title{
CRITICAL PROCESSES PRIORITIZATION IN A SANITARY COMPANY USING ANALYTIC HIERARCHY PROCESS
}

\begin{abstract}
This paper proposes a multi-criteria approach to identify the most relevant processes in a sanitary company that could affect continuity of service based on their legal impact, image, financial, environmental and social. Currently business continuity planning and contingency planning are elements of control that companies manage to determine the availability of their most critical processes in the event of an outage. Having a ranking of processes that may be more critical for the organization is vital for sustainable development and/or even the survival of any organization. A case study has been carried out in a sanitary company in Chile prioritizing critical processes that could allow develop an investment policy among other benefits. We have considered five business impacts as essential to be compared for each of the eight critical processes that possesses the organization. For the critical processes prioritization expert point of views, empirical data and by applying AHP the results are released, allowing enterprise to generate an investment plan and risk mitigation.
\end{abstract}

Keywords: AHP, Impact, Critical Process, Sanitary Company.

\section{Introduction}

Today in business there is growing a strong interest in having plans whose main objective is to protect the critical business processes, the aim of this initiative is to reduce the impacts to which is exposed the company because of closing one or more of these processes. Depending on the cause causing an interruption of the process is that the company risks financially, in the legal, environmental, social and even a topic of reputation.

That is why identifying critical processes through the assessment of impacts to which is exposed sanitary company will reveal the main threats to the organization and build capacity to mitigate them. The latter will make way for the organization to a strategy of medium and long term to continue the service and have more control if they suffer any interruption of critical processes. In this scenario, it is necessary to determine which are the most important processes for the company under study and thus address the actions to make improvements in those showing greater criticality. 


\section{Research Design/Methodology}

The initial approach begins with i) Generation of a rating scale for each impact (Legal, Image, Financial, Environmental and Social) comprising attributes for each process of the company (Production, Transportation, Distribution and Stock, Drainage, Depuration, Invoicing, Collection and Cobranza, Expansion, Contact Center) and ii) the design of the hierarchical structure of processes where critical categories for each level and relationships are obtained from the consulting experts.

The hierarchical structure to determine the prioritization criteria impact on business processes is designed with three levels where:

- Level 0: Indicates the target, the main objective: "Prioritization of the most critical processes in the Sanitary Company"

- Level 1: Includes impact criteria that will determine which process is more critical in the business.

- Level 2: Corresponds to the type of process in this case is eight independent processes, which means that there will be an analysis for each of them.

\section{Data/Model Analysis/Conclusions}

Applying the AHP of Saaty $(1997,1998)$ methodology and supported by Expert Choice (Saaty, 1983) concerning classification software is obtained. The results revealed that the priority social impacts is a determining factor in deciding which process should be prioritized in terms of investments in order to ensure the maximum operational continuity. For this particular case, the "social" impact had the highest priority (39.0\%), followed by "Picture" (19.0\%), "Financial" (17.0\%), "Environmental" (16.0\%) and finally "Legal" (9.0\%).

From these results, we can recognize that the main importance focuses on social and image impacts that could result in the company if a process stops operating.

The results obtained through AHP technologies are validated by the expert group and the prioritization of criteria showed low inconsistency.

The most critical process is Production (Social 27.0\%, Image 20.0\%, Financial 24.0\%, Environmental 19.0\%, Legal 18.0\%) followed by Drainage (Social 23.0\%, Image 19.0\%, Financial 8.0\%, Environmental 23.0\%, Legal 19.0\%), Depuration (Social 13,0\%, Image 8.0\%, Financial 20.0\%, Environmental 30.0\%, Legal 25.0\%), Distribution and Stock (Social 16.0\%, Image 15.0\%, Financial 11.0\%, Environmental 11.0\%, Legal 13.0\%), Transport (Social 15.0\%, Image 10.0\%, Financial 12.0\%, Environmental 12.0\%, Legal 8.0\%), Contact Center (Social 2.0\%, Image 24.0\%, Financial 4.0\%, Environmental 2.0\%, Legal 11.0\%), Invoicing, Collection and Cobranza (Social 2.0\%, Image 3.0\%, Financial 18.0\%, Environmental 2.0\%, Legal 4.0\%) and Expansion (Social 2.0 \%, Image 1.0\%, Financial 2.0\%, Environmental 2.0\%, Legal 3.0\%).

The results of this study can be considered in requiring water companies to assess which critical processes should be prioritized within an investment plan and risk mitigation, seeking to improve, to continue the operation and increase the accountability of the company. 